\title{
Chemo-dynamical evolution model: Enrichment of $r$-process elements in the Local Group dwarf galaxies
}

\author{
Yutaka Hirai $^{1,2,3}$, Yuhri Ishimaru ${ }^{4,5}$, Takayuki R. Saitoh ${ }^{6}$, \\ Michiko S. Fujii ${ }^{2}$, Jun Hidaka ${ }^{7,2}$ and Toshitaka Kajino ${ }^{2,1}$ \\ ${ }^{1}$ Department of Astronomy, Graduate School of Science, The University of Tokyo, \\ 7-3-1 Hongo, Bunkyo-ku, Tokyo 113-0033, Japan \\ email: yutaka.hirai@nao.ac.jp \\ ${ }^{2}$ Division of Theoretical Astronomy, National Astronomical Observatory of Japan, \\ 2-21-1 Osawa, Mitaka, Tokyo 181-8588, Japan \\ ${ }^{3}$ Research Fellow of Japan Society for the Promotion of Science \\ ${ }^{4}$ Department of Natural Sciences, International Christian University, \\ 3-10-2 Osawa, Mitaka, Tokyo 181-8585, Japan \\ ${ }^{5}$ Institut d'Astrophysique de Paris, \\ 98bis Boulevard Arago, 75014, Paris, France \\ ${ }^{6}$ Earth-Life Science Institute, Tokyo Institute of Technology, \\ 2-12-1 Ookayama, Meguro-ku, Tokyo 152-8551, Japan \\ ${ }^{7}$ School of Science and Engineering, Meisei University, \\ 2-1-1 Hodokubo, Hino, Tokyo 191-0042, Japan
}

\begin{abstract}
Neutron star mergers are one of the candidate astrophysical site(s) of $r$-process. Several chemical evolution studies however pointed out that the observed abundance of $r$-process is difficult to reproduce by neutron star mergers. In this study, we aim to clarify the enrichment of $r$-process elements in the Local Group dwarf galaxies. We carry out numerical simulations of galactic chemo-dynamical evolution using an $N$-body/smoothed particle hydrodynamics code, ASURA. We construct a chemo-dynamical evolution model for dwarf galaxies assuming that neutron star mergers are the major source of $r$-process elements. Our models reproduce the observed dispersion in $[\mathrm{Eu} / \mathrm{Fe}]$ as a function of $[\mathrm{Fe} / \mathrm{H}]$ with neutron star mergers with a merger time of 100 Myr. We find that star formation efficiency and metal mixing processes during the first $\lesssim 300$ Myr of galaxy evolution are important to reproduce the observations. This study supports that neutron star mergers are a major site of $r$-process.
\end{abstract}

Keywords. galaxies: abundances — galaxies: dwarf — galaxies: evolution — Local Group — methods: numerical

Neutron star mergers (NSMs) are one of the promising astrophysical sites of $r$-process. Argast et al. (2004) suggested that it is difficult to reproduce the observed star-to-star scatters in extremely metal-poor stars due to the long merger time ( $100 \mathrm{Myr})$ and low rate $\left(\sim 10^{-4} \mathrm{yr}^{-1}\right.$ for a Milky Way size galaxy) of NSMs. On the other hand, Ishimaru et al. (2015) pointed out that this problem can be solved if the Milky Way halo was formed from sub-haloes with low star formation efficiency. Enrichment of $r$-process elements (e.g., Eu) in dwarf galaxies which would be the building blocks of the Milky Way is not understood yet. We aim to clarify the enrichment of $r$-process elements in dwarf galaxies with a high-resolution chemo-dynamical evolution model assuming NSMs are the major site of $r$-process. 


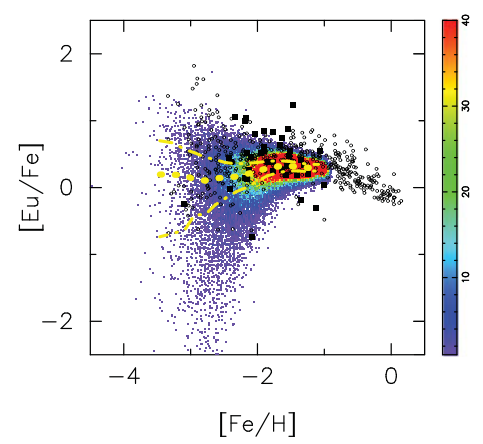

Figure 1. $[\mathrm{Eu} / \mathrm{Fe}]$ as a function of $[\mathrm{Fe} / \mathrm{H}]$. Different shades of grey refer to the number of stars produced in our model (legend on the right). The dotted curve is the median of the model predictions. The dash-dotted curves are the first and third quartiles, respectively. Circles are observed values for Galactic halo stars (SAGA database, Suda et al. 2008). Squares are the observed value of stars in Carina, Draco, Leo I, Sculptor, and Ursa Minor dSphs (SAGA database, Suda et al. 2014).

We perform a series of simulations using an $N$-body/smoothed particle hydrodynamics code, ASURA (Saitoh et al. 2008; Saitoh et al. 2009). This code includes metallicity dependent cooling, star formation, and supernova feedback. We adopt metal mixing assuming the average metallicity of 32 surrounding gas particles to a newly formed star particle. For dwarf galaxy models, both dark matter and gas particles are initially distributed along with the pseudo isothermal profile following Revaz \& Jablonka (2012). Details of implementation to the code and models are discussed in Hirai et al. (2015).

Figure 1 shows $[\mathrm{Eu} / \mathrm{Fe}]$ as a function of $[\mathrm{Fe} / \mathrm{H}]$ in our model assuming NSMs with a merger time of $100 \mathrm{Myr}$. As shown in this figure, star-to-star scatters of $[\mathrm{Eu} / \mathrm{Fe}]$ in extremely metal-poor stars produced by NSMs with a merger time of $100 \mathrm{Myr}$ are consistent with the observations. This is because the average metallicity of stars is constant during the first $\sim 300$ Myr from the beginning of the star formation in the galaxy. Due to the low star formation efficiency of the galaxy, the spatial distribution of metallicity is highly inhomogeneous in the first $\lesssim 300$ Myr. Since most gas particles are enriched by a single supernova in this epoch, the metallicity of the stars is mainly determined by the distance from each supernova to the gas particles, which formed the stars. NSMs with merger time of $100 \mathrm{Myr}$ can therefore account for the observations of extremely metal-poor stars. In contrast, metallicity is well correlated with the galactic age after $\sim 300 \mathrm{Myr}$, irrespective of the distance from each supernova to the gas particle. Since supernova products have already been well mixed in a galaxy, the stellar metallicity is determined by the number of supernovae.

\section{References}

Argast, D., Samland, M., Thielemann, F.-K., \& Qian, Y.-Z. 2004, A\& A, 416, 997

Hirai, Y., Ishimaru, Y., Saitoh, T. R., Fujii, M. S., Hidaka, J., \& Kajino, T. 2015, ApJ, 814, 41 Ishimaru, Y., Wanajo, S., \& Prantzos, N. 2015, ApJ, (Letter), 804, L35

Revaz, Y. \& Jablonka, P. 2012, A\&AA, 538, A82

Saitoh, T. R., Daisaka, H., Kokubo, E., Makino, J., Okamoto, T., Tomisaka, K., Wada, K., \& Yoshida, N. 2008, PASJ, 60, 667

Saitoh, T. R., Daisaka, H., Kokubo, E., Makino, J., Okamoto, T., Tomisaka, K., Wada, K., \& Yoshida, N. 2009, PASJ, 61, 481

Suda, T., Katsuta, Y., Yamada, S., et al. 2008, PASJ, 60, 1159

Suda, T., Hidaka, J., \& Ishigaki, M., et al. 2014, MmSAI, 85, 600 